\section{Informant Questionnaire on Cognitive Decline in the Elderly (IQCODE): adaptação transcultural para uso no Brasil}

\author{
Informant Questionnaire on Cognitive Decline \\ in the Elderly (IQCODE): cross-cultural \\ adaptation for use in Brazil
}

\footnotetext{
${ }^{1}$ Faculdade de Ciências Médicas, Universidade do Estado do Rio de Janeiro, Rio de Janeiro, Brasil.

Correspondência M. A. S. Sanchez Departamento de Pós-graduação, Faculdade de Ciências Médicas, Universidade do Estado do Rio de Janeiro. Av. Marechal Rondon 381, 2o andar, Rio de Janeiro, $R J$ 20950-000, Brasil. asanchezrj@terra.com.br
}

\begin{abstract}
This article presents the results of the translation and cross-cultural adaptation of the Informant Questionnaire on Cognitive Decline in the Elderly (IQCODE) and the test-retest reliability of a version to be used in Brazil. An instrument review committee analyzed the conceptual and item equivalence. In order to analyze semantic equivalences, three translations and three backtranslations were performed; a summary version was devised and pre-tested, and a test version elaborated. The Cronbach's $\alpha$ coefficient and intraclass correlation coefficient (ICC) were used to measure the internal consistency and test-retest reliability, respectively. Of the 169 informants assessed, 97 were retested. A Cronbach's $\alpha$ of 0.94 and an ICC of 0.92 were found. The reliability levels lead to the conclusion that the IQCODE-BR version is easy to comprehend. Satisfactory equivalence to the original version was observed.
\end{abstract}

Questionnaires; Translating; Dementia
Maria Angélica dos Santos Sanchez ${ }^{1}$ Roberto Alves Lourenço ${ }^{1}$

\section{Introdução}

No Brasil, ainda não dispomos de dados precisos acerca da prevalência da demência no país, embora alguns estudos, de localização geográfica limitada, tenham apontado freqüências variáveis, em grande parte por causa das distintas metodologias empregadas 1,2,3. Tais trabalhos esbarraram em problemas metodológicos, dentre os quais nos interessa apontar a falta de um relato padronizado do informante para o diagnóstico da síndrome demencial.

Não obstante, cresce a importância de se planejar ações que possam identificar o indivíduo com problemas cognitivos. Geralmente, os instrumentos, utilizados para o rastreio do declínio cognitivo têm baixa acurácia em determinadas condições, como nas fases iniciais dos quadros demenciais, em idades avançadas, na presença de baixa escolaridade e déficits sensoriais, e diante das várias desordens mentais 4 .

A baixa escolaridade é um dos problemas que mais interferem nos resultados dos testes cognitivos e, no Brasil, o analfabetismo é, não raro, uma questão relevante; segundo o último censo demográfico nacional, $35,2 \%$ da população com mais de 60 anos são analfabetos 5 .

Por outro lado, pela forma insidiosa do aparecimento do quadro demencial e pela progressão habitualmente lenta que caracteriza a maior parte das morbidades que levam à demência, ela 
parece não ser adequadamente avaliada e diagnosticada na sua fase inicial.

As iniciativas assistenciais na política de saúde, provendo suporte medicamentoso para o idoso com diagnóstico de doença de Alzheimer, ganham relevância, sobretudo por alcançar uma parcela menos privilegiada financeiramente cujo acesso a esta terapêutica somente é possível por meio de políticas públicas. No entanto, a dificuldade de detectar a doença, comumente, faz que ela seja diagnosticada em estágios avançados.

Há um razoável consenso entre especialistas em torno dos critérios utilizados para o diagnóstico da síndrome demencial: a presença de déficit de memória, associado a alterações em pelo menos uma outra área cognitiva (afasia, agnosia, apraxia, alterações de funções executivas); a presença de prejuízos funcional e ocupacional relacionados ao déficit cognitivo; e os problemas cognitivos não devem ocorrer exclusivamente no curso de um delirium. A realização de avaliação neuropsicológica objetiva e o relato de um informante confirmando as perdas cognitivas e funcionais acima mencionadas são elementos fundamentais no algoritmo de decisão diagnóstica 6 .

Entre os inúmeros testes cognitivos habitualmente utilizados no rastreamento e diagnóstico desta síndrome, o Mini-Exame do Estado Mental (MEEM) 7 é um dos mais citados na literatura nacional e internacional. Entretanto, sofre a influência da escolaridade, da idade e das habilidades pré-mórbidas ${ }^{8}$. Por isso mesmo, alguns estudos foram conduzidos de forma a combinar o MEEM com um relato do informante para aumentar a acurácia do diagnóstico 4,8,9,10,11,12,13.

O relato de um informante confiável como parte fundamental dos critérios estabelecidos para o diagnóstico da síndrome demencial 6,14,15 vem sendo, nos últimos anos, largamente estudado em outros países.

Os instrumentos com base no relato do informante permitem avaliações longitudinais, mostram as alterações no desempenho cognitivo e funcional dos indivíduos e podem ser utilizados quando da impossibilidade dos sujeitos realizarem testes cognitivos 16,17,18,19.

A maioria deles, não obstante as limitações relacionadas à disponibilidade de uma pessoa adequada, apresentam as seguintes vantagens: avaliam o declínio cognitivo com base nas demandas impostas pelo ambiente em que o indivíduo está inserido; podem ser realizados mesmo na ausência do indivíduo com suspeita de declínio cognitivo; podem ser feitos ainda que o paciente esteja muito comprometido ou instável; podem ser conduzidos por carta ou telefone; podem ser realizados em diversos contextos culturais 20 .
Dentre as entrevistas padronizadas para este fim, o Informant Questionnaire on Cognitive Decline in the Elderly (IQCODE) é um instrumento largamente utilizado. Trata-se de um questionário para detecção do declínio cognitivo com base no relato do informante, que foi desenvolvido na Austrália, no idioma Inglês, composto, em sua versão original, por 26 itens. As perguntas estão organizadas em uma escala Likert, com cinco opções: 1 - muito melhor; 2 - um pouco melhor; 3 - não houve mudança; 4 - um pouco pior; 5 - muito pior.

O resultado final é obtido pela soma ponderada dos itens, dividindo-os pelo total de itens da escala. O escore varia de um a cinco; os escores menores ou iguais a três indicam que não está havendo alteração, igual a quatro indica uma considerável alteração, e igual a cinco indica muita alteração. O ponto de corte obtido no estudo original foi de 3,27/3,30 21. Jorm \& Korten 21, em 1988, propõem que ele seja aplicado em parente ou amigo próximo que conviva com o idoso há pelo menos dez anos, com o objetivo de comparar as alterações no desempenho do indivíduo durante este período de convivência.

Duas outras versões do instrumento são apresentadas: uma reduzida, composta por 16 itens validada por Jorm ${ }^{22}$, em 1994, e outra, o IQCODE retrospectivo, que é uma variação do instrumento, elaborada por Thomas et al. 23, em 1994, para ser aplicada ao informante após a morte do idoso.

O IQCODE foi adaptado para diversos contextos culturais. Compartilha as mesmas vantagens com as demais entrevistas que utilizam a estratégia de buscar informações cognitivas junto a um informante próximo e, como sugerido por alguns trabalhos, não sofre influências substanciais de idade e escolaridade 16,18,20,21,24,25.

Os estudos realizados com o IQCODE trazem informações substanciais acerca do rastreio e diagnóstico da síndrome demencial mesmo na fase inicial, e a escala tem sido indicada como um excelente recurso para estes fins, sobretudo pelo fato de parecer não sofrer influência das habilidades pré-mórbidas, como ocorre na maioria dos testes neuropsicológicos 16,17,18,19,20,21,24,25.

O IQCODE se mostrou também um excelente recurso para o rastreio da síndrome demencial em estudos comunitários 11,19,25, o que parece fazer dele um instrumento de grande valia para a identificação de problemas cognitivos em vários níveis de atenção.

No Brasil, ainda são poucos os estudos acerca dos instrumentos que avaliam o declínio cognitivo com base no relato do informante. Os trabalhos realizados apenas os utilizaram como ferramenta complementar no diagnóstico da de- 
mência 4,9,10, mas não tiveram o intuito de avaliarem as suas propriedades psicométricas, de incluí-los na atenção à saúde do idoso, nem de torná-los parte de propostas de planejamento de ações de rastreio do declínio cognitivo em populações.

Mesmo sem um processo formal de adaptação, os estudos conduzidos no Brasil chamaram a atenção para a necessidade de incluirmos, nos vários níveis de atenção, instrumentos padronizados que possam, a partir do relato do informante, fornecer dados que auxiliem no rastreio ou no diagnóstico da síndrome demencial.

Apesar de a utilização do mesmo ter apresentado uma boa aceitação em nossa cultura, ainda carecíamos de estudos que avaliassem as características de uma versão que tivesse sido submetida a um processo formal de adaptação para o nosso contexto.

O presente estudo teve como objetivo a adaptação transcultural da versão original do IQCODE e a determinação da confiabilidade teste-reteste da versão obtida para uso no Brasil.

\section{Metodologia}

A seleção do questionário resultou de uma revisão de instrumentos de avaliação do declínio cognitivo em idosos, com base no relato de familiares ou amigos próximos. O IQCODE foi escolhido por ser uma escala breve, com uma duração média de dez minutos de aplicação, de fácil administração e que permite estudos longitudinais para avaliação do declínio, ao contrário de outras entrevistas que, em geral, avaliam o estado atual do indivíduo.

\section{Delineamento, local do estudo e amostra}

É um estudo de corte transversal, cuja população fonte foi composta por 1.200 indivíduos assistidos no ambulatório de geriatria de um hospital universitário na cidade do Rio de Janeiro. Foi selecionada uma amostra de conveniência composta de 169 acompanhantes de idosos que foram consultados no ambulatório no período de 2 de abril a 15 de dezembro de 2006.

Os critérios de inclusão para o idoso foram: estar em acompanhamento no ambulatório; ser brasileiro; ter 65 anos ou mais; ter sido submetido à avaliação geriátrica ampla para ingresso no serviço; ter tido sua última consulta, com avaliação funcional e cognitiva, há menos de um ano. Foram incluídos os informantes cuja convivência com estes idosos fosse igual ou superior a dez anos e fossem conhecedores do desempenho dos idosos nas atividades de vida diária.
Foram excluídos os idosos portadores de patologias psiquiátricas; aqueles com deficiência visual e auditiva grave e comprometimento psicomotor; com demência em estágio avançado e/ou com resultado $\leq 13$ no MEEM 7,26. Os informantes que apresentavam relato de conflitos com o idoso ou importante deficiência auditiva e visual também foram excluídos do estudo.

\section{Adaptação transcultural do IQCODE}

O roteiro de adaptação deste estudo foi baseado na proposta de Herdman et al. 27 , que recomendam a avaliação das equivalências conceitual e de itens; semântica; operacional; de mensuração e equivalência funcional. $\mathrm{O}$ modelo admite ser possível adaptar instrumentos para outras culturas, porém se faz necessária a avaliação da equivalência entre os construtos. Para tanto, sugerem alguns cuidados até que surja uma nova versão de determinado instrumento.

Para adaptação do IQCODE à cultura brasileira foram percorridas algumas das etapas sugeridas pelos autores 27 .

\section{- Equivalência conceitual e de itens}

Nesta etapa, após a escolha do instrumento, foi realizada uma minuciosa revisão sobre seu uso. Obteve-se uma versão traduzida informalmente no Brasil que foi aplicada a aproximadamente 100 acompanhantes de idosos assistidos em uma unidade ambulatorial geriátrica, com o objetivo de observar como a população-alvo reagia frente a este tipo de avaliação. Esta primeira etapa mostrou que o instrumento era de fácil administração, com boa aceitação; no entanto requeria ajustes, visto que alguns itens não eram facilmente compreendidos. Foi em razão das impressões de desempenho do instrumento, quando usado em serviço, que se optou por um processo formal de adaptação transcultural.

Formou-se um comitê de revisão com um grupo de especialistas composto por cinco profissionais que atuam na área da geriatria e gerontologia que - com base no conhecimento prévio do instrumento, por intermédio da literatura - analisou todos os itens que compunham o original, explorando se suas diferentes dimensões eram pertinentes para uso na cultura brasileira.

\section{- Equivalência semântica}

Foram realizadas três traduções independentes para o português. As retrotraduções para o inglês foram realizadas, independentes, por dois ingleses e um americano, com fluência nos dois idiomas e radicados no Brasil há 15 anos. Na se- 
qüência, um professor de inglês-literaturas, brasileiro, bilíngüe, que desconhecia a procedência das retrotraduções, elaborou uma versão-síntese, valendo-se da comparação entre as retrotraduções e o instrumento original. Posteriormente, o comitê de revisão reuniu-se para análise da versão proposta.

O pré-teste foi realizado pelo primeiro autor do trabalho. Nesta etapa, era solicitado ao entrevistado que parafraseasse cada item para que o entrevistador pudesse avaliar a compreensão. Os sinônimos utilizados pelo grupo que compreendia bem todos os itens eram destacados de forma que pudessem ser aproveitados em uma nova versão-teste.

O comitê de revisão realizou ajustes semânticos sendo, então, elaborada uma segunda edição da versão-teste. Esta versão foi aplicada a outros 15 informantes. Da mesma forma anterior, era solicitado aos entrevistados que parafraseassem cada item. Com a aplicação dessa versão não foram observados problemas e se iniciou o estudo piloto.

\section{- Equivalência operacional}

O instrumento original foi concebido para ser autopreenchível; todavia estudos subseqüentes à sua elaboração o utilizaram mediante entrevista direta, por carta e por telefone. Para o presente estudo, optou-se por entrevista face a face, já que não conhecíamos com anterioridade o nível de instrução dos entrevistados, e considerávamos que esta variável poderia se constituir em um viés de aferição. A estrutura do questionário e as instruções sugeridas pelo autor do instrumento foram mantidas.

\section{- Equivalência de mensuração}

Dentro de um programa de investigação que deverá, em uma próxima etapa, determinar diferentes aspectos da validade do instrumento, avaliou-se a sua estabilidade no tempo pelo cálculo da confiabilidade teste-reteste.

\section{Procedimentos estatísticos}

Para armazenamento e análise dos dados foi utilizado o pacote estatístico SPSS, versão 12.0 (SPSS Inc., Chicago, Estados Unidos). Foram avaliadas as freqüências descritivas para os dados sócio-demográficos dos idosos e dos informantes A consistência interna do questionário foi mensurada pelo coeficiente alpha de Cronbach, e a confiabilidade teste-reteste pelo coeficiente de correlação intraclasse (CCI) para o escore do questionário.

\section{Procedimentos para seleção da amostra}

Duas médicas revisavam os prontuários dos idosos com consulta agendada e selecionavam os que apresentavam os critérios de elegibilidade para participar do estudo. Todo sujeito elegível era contatado via telefone com o objetivo de se identificar um acompanhante que pudesse colaborar como informante. A estes acompanhantes eram fornecidas informações breves acerca do estudo e, em seguida, eram convidados a comparecer no ambulatório no dia da consulta.

A cada dia de consulta, um supervisor das atividades de campo captava, na sala de espera, os idosos previamente contatados, que estivessem com acompanhantes. O idoso e o acompanhante eram conduzidos a uma sala onde era explicado todo o objetivo do estudo. O aceite era considerado quando ambos concordavam em participar. Em seguida o termo de consentimento livre e esclarecido era lido e explicado e eram coletados os dados sócio-demográficos do informante. Depois, o informante era apresentado ao auxiliar de pesquisa e encaminhado a uma sala reservada para responder a entrevista.

\section{Procedimentos para aplicação da versão teste do IQCODE-BR}

Cinco auxiliares de pesquisa foram treinados durante duas semanas, perfazendo um total de 16 horas. O treinamento consistia na leitura do manual de instruções e simulação das entrevistas. A cada entrevista eram realizados os ajustes para padronizar a coleta de dados.

Os auxiliares iniciaram o estudo piloto que foi realizado durante vinte dias com a aplicação de quarenta questionários para analisar a estrutura operacional. Nessa fase, todas as entrevistas foram gravadas e, posteriormente, ouvidas pelo primeiro autor com o objetivo de avaliar o desempenho dos auxiliares de pesquisa. Neste piloto não foram observados problemas, sendo mantida a estrutura proposta. Os indivíduos que aceitaram participar do estudo foram avaliados pela versão-teste do IQCODE-BR (Tabela 1).

A avaliação da psicometria do instrumento percorrerá um longo trajeto até que se possa avaliar a equivalência funcional. Contudo, optouse por uma avaliação preliminar para analisar a compreensão pela população alvo para que se pudesse, então, dar continuidade às análises subseqüentes.

A confiabilidade teste-reteste foi analisada pela reaplicação do instrumento pelo mesmo aferidor em nova entrevista, e todos os informantes foram convidados a participar. Os entrevistadores desconheciam o diagnóstico cognitivo do idoso. 
"Gostaria que o(a) Senhor(a) recordasse o estado em que o $\operatorname{Sr}(a)$ X se encontrava há dez anos, em 19_, e o comparasse com seu estado atual. Descrevemos abaixo situações em que ele(a) tenha de usar a memória ou o raciocínio e eu gostaria que o(a) Sr(a) dissesse se, nesse aspecto, ele(a) melhorou, piorou, ou permaneceu na mesma nos últimos 10 anos. É muito importante comparar o desempenho atual do $\operatorname{Sr}(\mathrm{a}) \mathrm{X}$ com o de 10 anos atrás. Deste modo, se há dez anos ele(a) sempre se esquecia onde havia deixado as coisas e isso ainda acontece, então isto será considerado como "POUCA MUDANÇA". Diga-me, a seguir as mudanças que o(a) Senhor(a) observou, apontando no cartão a melhor resposta para cada item. Comparado com seu estado há dez anos, com está o Sr(a) X para...".

\begin{tabular}{|c|c|c|c|c|c|}
\hline Itens & $\begin{array}{l}\text { Muito } \\
\text { melhor }\end{array}$ & $\begin{array}{l}\text { Um pouco } \\
\text { melhor }\end{array}$ & $\begin{array}{c}\text { Pouca } \\
\text { mudança }\end{array}$ & $\begin{array}{l}\text { Um pouco } \\
\text { pior }\end{array}$ & $\begin{array}{c}\text { Muito } \\
\text { pior }\end{array}$ \\
\hline 1. Lembrar de rostos de parentes e amigos & 1 & 2 & 3 & 4 & 5 \\
\hline 2. Lembrar dos nomes de parentes e amigos & 1 & 2 & 3 & 4 & 5 \\
\hline \multicolumn{6}{|l|}{ 3. Lembrar de fatos relacionados a parentes e amigos como, } \\
\hline por exemplo: suas profissões, aniversários e endereços & 1 & 2 & 3 & 4 & 5 \\
\hline 4. Lembrar de acontecimentos recentes & 1 & 2 & 3 & 4 & 5 \\
\hline 5. Lembrar de conversas depois de poucos dias & 1 & 2 & 3 & 4 & 5 \\
\hline 6. No meio de uma conversa, esquecer o que ele(a) queria dizer & 1 & 2 & 3 & 4 & 5 \\
\hline 7. Lembrar do próprio endereço e telefone & 1 & 2 & 3 & 4 & 5 \\
\hline 8. Saber o dia, o mês em que estamos & 1 & 2 & 3 & 4 & 5 \\
\hline 9. Lembrar onde as coisas são geralmente guardadas & 1 & 2 & 3 & 4 & 5 \\
\hline \multicolumn{6}{|l|}{ 10. Lembrar onde encontrar coisas que foram guardadas em } \\
\hline lugares diferentes daqueles em que costuma guardar & 1 & 2 & 3 & 4 & 5 \\
\hline 11. Adaptar-se a qualquer mudança no dia-a-dia & 1 & 2 & 3 & 4 & 5 \\
\hline 12. Saber utilizar aparelhos domésticos & 1 & 2 & 3 & 4 & 5 \\
\hline 13. Aprender a utilizar um novo aparelho existente na casa & 1 & 2 & 3 & 4 & 5 \\
\hline 14. Aprender coisas novas em geral & 1 & 2 & 3 & 4 & 5 \\
\hline 15. Lembrar das coisas que aconteceram na juventude & 1 & 2 & 3 & 4 & 5 \\
\hline 16. Lembrar de coisas que ele(a) aprendeu na juventude & 1 & 2 & 3 & 4 & 5 \\
\hline 17. Entender o significado de palavras pouco utilizadas & 1 & 2 & 3 & 4 & 5 \\
\hline 18. Entender o que é escrito em revistas e jornais & 1 & 2 & 3 & 4 & 5 \\
\hline 19. Acompanhar histórias em livros ou em programas de televisão & 1 & 2 & 3 & 4 & 5 \\
\hline 20. Escrever uma carta para amigos ou com fins profissionais & 1 & 2 & 3 & 4 & 5 \\
\hline 21. Conhecer importantes fatos históricos & 1 & 2 & 3 & 4 & 5 \\
\hline 22. Tomar decisões no dia-a-dia & 1 & 2 & 3 & 4 & 5 \\
\hline 23. Lidar com dinheiro para as compras & 1 & 2 & 3 & 4 & 5 \\
\hline \multicolumn{6}{|l|}{ 24. Lidar com assuntos financeiros, por exemplo: } \\
\hline aposentadoria e conta bancária & 1 & 2 & 3 & 4 & 5 \\
\hline \multicolumn{6}{|l|}{ 25. Lidar com outros cálculos do dia-a-dia, por exemplo: } \\
\hline \multicolumn{6}{|l|}{ quantidade de comida a comprar, há quanto tempo não } \\
\hline recebe visitas de parentes ou amigos & 1 & 2 & 3 & 4 & 5 \\
\hline \multicolumn{6}{|l|}{ 26. Usar sua inteligência para compreender e pensar } \\
\hline sobre o que está acontecendo & 1 & 2 & 3 & 4 & 5 \\
\hline
\end{tabular}

O estudo recebeu autorização do autor, Anthony Jorm, e foi submetido à apreciação e aprovação pelo Comitê de Ética em Pesquisa do Hospital Universitário Pedro Ernesto. Conforme preconiza a Resolução no. 196/96 do Conselho Nacional de Saúde, todos os participantes autorizaram a participação no estudo mediante assinatura de termo de consentimento livre e esclarecido.

\section{Resultados}

Participaram do estudo 169 informantes, dos quais 97 retornaram para o reteste. Destes, $74,2 \%$ eram do sexo feminino. As idades variaram de 23 a 85 anos com a média de 57,5 anos (desvio-padrão - DP $=14,9)$. A renda média era de 2,9 salários ( $\mathrm{DP}=2,17)$. A escolaridade dos participantes 
variou de 0 a 12 anos, a média foi de 8,5 anos $(\mathrm{DP}=3,71) ; 64,9 \%$ eram casados (Tabela 2 ). Dentre os idosos, sujeitos do estudo, $66 \%$ eram do sexo feminino. A média de idade foi 77,7 anos (DP $=6,37)$ com escolaridade média de 4,3 anos $(\mathrm{DP}=3,6)$. Destes, 39,2\% com diagnóstico de síndrome demencial (Tabela 3). A aplicação do IQCODE-BR teve duração média de dez minutos (DP $=4,59)$.

Nas reuniões com o comitê de revisão, ficou claro que o construto cognição, embutido no conjunto de itens que compõem o instrumento, poderia ser perfeitamente transposto diretamente à cultura brasileira, em geral, e ao contexto particular do segmento social em teste, sem que para isso se necessitasse produzir adaptações por inexistência, divergência ou diferenças em qualquer grau significativo dos conceitos subjacentes. Concluiu-se que todos os itens que compunham o instrumento original apresentavam conceitos aplicáveis à cultura brasileira.

A consistência interna da versão do IQCODEBR mensurada pelo alpha de Cronbach foi de

Tabela 2

Características sócio-demográficas de uma amostra de informantes que participaram do estudo de adaptação transcultural do questionário do informante para detecção de declínio cognitivo em idosos, $2006(n=97)$

\begin{tabular}{|c|c|c|}
\hline Variáveis & Freqüência & $\%$ \\
\hline \multicolumn{3}{|l|}{ Gênero } \\
\hline Masculino & 25 & 25,8 \\
\hline Feminino & 72 & 74,2 \\
\hline \multicolumn{3}{|l|}{ Idade (anos) } \\
\hline 29-39 & 12 & 12,4 \\
\hline $40-49$ & 12 & 12,4 \\
\hline $50-59$ & 30 & 30,9 \\
\hline $60-69$ & 21 & 21,6 \\
\hline$\geq 70$ & 22 & 22,7 \\
\hline \multicolumn{3}{|l|}{ Estado conjugal } \\
\hline Casado & 63 & 64,9 \\
\hline Solteiro & 19 & 19,6 \\
\hline Viúvo & 7 & 7,2 \\
\hline Divorciado & 8 & 8,3 \\
\hline \multicolumn{3}{|c|}{ Renda mensal (salários mínimos) } \\
\hline Sem renda própria & 18 & 18,5 \\
\hline $1-3$ & 48 & 49,5 \\
\hline $4-6$ & 19 & 19,6 \\
\hline$\geq 7$ & 12 & 12,4 \\
\hline \multicolumn{3}{|l|}{ Escolaridade (anos) } \\
\hline Analfabetos & 2 & 2,1 \\
\hline $1-4$ & 20 & 20,6 \\
\hline $5-8$ & 21 & 21,6 \\
\hline$\geq 9$ & 54 & 55,7 \\
\hline
\end{tabular}

0,94 . Nas entrevistas reaplicadas entre 14 e 60 dias, média de 28 dias, obteve-se um CCI de 0,92.

\section{Discussão}

Historicamente, no Brasil, assim como em outros países, o processo de adaptação de instrumentos vem se apoiando apenas na simples tradução do original, sem o uso de uma metodologia adequada que leve em consideração as peculiaridades da população-alvo. Para que haja uma mudança nessa prática, faz-se necessário que além da tradução sejam considerados os ajustes locais e regionais e, tal trabalho requer rigor na adaptação de instrumentos de aferição oriundos de outros contextos culturais 28 .

Embora de forma lenta, está crescendo no Brasil o número de estudos que realizam adaptação transcultural, seguindo uma metodologia adequada. Tais estudos são observados especialmente na área de saúde mental, em que escalas e testes cognitivos vêm sendo submetidos a um rigoroso processo de adaptação.

A adaptação cultural do IQCODE, ao se alicerçar na metodologia da avaliação das equivalências 27,29 , buscou imprimir qualidade a uma versão para ser estudada no contexto brasileiro.

Neste trabalho, realizado com perspectiva universalista 27 , procurou-se seguir ao máximo a tradução literal, respeitando, porém, as características da população em que foi testada.

No que concerne à avaliação semântica, no primeiro pré-teste com o IQCODE-BR foram observados pequenos problemas de compreensão por parte de alguns entrevistados. Foram sugeridas alterações consideradas pertinentes. Além dessas alterações, foi também elaborado um manual de instruções indicando as possibilidades de exemplos de cada item. Isto foi importante para evitar a interpretação não padronizada do entrevistador, fato que poderia ocasionar vieses de aferição.

As alterações propostas no manual foram importantes em alguns casos como, por exemplo, nos itens 4 e 5 (Tabela 4), quando se optou por estabelecer tempo para delimitar o período referente a acontecimentos recentes (últimos três meses), e lembrar de conversas depois de poucos dias (últimos três dias). A preocupação foi diferenciar os vocábulos "recentes" de "poucos dias".

Da mesma maneira, nos itens 9 e 10 (Tabela 4), escolheu-se distinguir os objetos que possuem um lugar fixo daqueles que são deixados ocasionalmente em quaisquer outros espaços da casa. O informante, em geral, considerava uma atitude normal esquecer onde coloca os óculos ou chaves. Ao exemplificar os objetos que pos- 
suem um lugar fixo como as roupas e os talheres a intenção foi obter informação adequada acerca da orientação espacial.

Outra inserção que parece ter acrescentado maior entendimento, diz respeito ao item 19, onde se incluiu assistir à novela junto das opções acompanhar uma historia em um livro ou na TV. Este é um forte hábito na nossa cultura, e foi observado como um importante marcador de mudança de comportamento no relato do informante. Isso pode estar relacionado ao fato de a novela ser apresentada diariamente, com pelo menos três diferentes histórias por dia, sendo facilmente notadas as dificuldades de entendimento das situações apresentadas.

O alpha de Cronbach $(0,94)$ foi similar aos achados originais apresentados por Jorm \& Korten ${ }^{21}$, sugerindo que o processo adaptação a que foi submetido não alterou a consistência dos seus itens. Em outros estudos também foram observados valores semelhantes como o de Jorm \& Jacomb 25 que encontraram um alpha de 0,95. O trabalho de Fuh et al. 30 apresentou um alpha de 0,97 e o de Tang et al. 31, um alpha de 0,95. Como no Brasil não foram encontrados estudos que avaliassem as características psicométricas deste instrumento, nem de outro semelhante, não foi possível estabelecer comparações no âmbito do nosso contexto cultural.

O período estipulado entre o teste e o reteste - 14 a 60 dias - parece não afetar a interpretação do informante quanto ao comportamento geral do idoso, pois o curso da maior parte das patologias demenciantes - por exemplo, a demência do tipo Alzheimer - parece não sofrer alterações em um curto período, a não ser por um problema agudo, fato não relatado por nenhum informante.

Em geral, os estudos que avaliaram o desempenho do IQCODE trazem as análises de correlações com outros instrumentos de rastreio da demência. Apesar do grande número de estudos de validação do IQCODE em outras culturas, são poucas as informações sobre as avaliações da estabilidade e reprodutibilidade da escala.

Uma das limitações deste desenho de estudo foi o grande número de perdas $(\mathrm{n}=72)$. Como o informante não é a pessoa acompanhada na unidade de saúde, o retorno para o reteste não aconteceu com a freqüência esperada. Os idosos mais saudáveis do ponto de vista funcional, freqüentemente, comparecem à consulta sem acompanhantes. Por outro lado, questões como a inserção do informante no mercado de trabalho, custos de deslocamento e impossibilidade de deixar o idoso em casa, também foram responsáveis pela não-participação em um segundo momento da pesquisa; entretanto tais perdas não influenciaram os resultados obtidos.
Tabela 3

Características sócio-demográficas da amostra de idosos que participaram do estudo de adaptação transcultural do questionário do informante para detecção de declínio cognitivo em idosos, 2006 ( $n=97)$.

\begin{tabular}{|c|c|c|}
\hline Variáveis & Freqüência & $\%$ \\
\hline \multicolumn{3}{|l|}{ Gênero } \\
\hline Masculino & 33 & 34 \\
\hline Feminino & 64 & 66 \\
\hline \multicolumn{3}{|l|}{ Idade (anos) } \\
\hline $65-69$ & 12 & 12,4 \\
\hline $70-74$ & 16 & 16,5 \\
\hline $74-79$ & 32 & 33 \\
\hline $80-85$ & 37 & 38,1 \\
\hline \multicolumn{3}{|c|}{ Escolaridade (anos) } \\
\hline Analfabeto & 17 & 17,5 \\
\hline $1-4$ & 48 & 49,5 \\
\hline $5-8$ & 19 & 19,6 \\
\hline$\geq 9$ & 13 & 13,4 \\
\hline \multicolumn{3}{|c|}{ Síndrome demencial * } \\
\hline Sim & 34 & 35,1 \\
\hline Não & 66 & 64,9 \\
\hline \multicolumn{3}{|l|}{ MEEM ** } \\
\hline $14-18$ & 27 & 27,8 \\
\hline $19-23$ & 29 & 29,9 \\
\hline$\geq 24$ & 41 & 42,3 \\
\hline \multicolumn{3}{|l|}{ Depressão *** } \\
\hline Sim & 38 & 39,2 \\
\hline Não & 59 & 60,8 \\
\hline
\end{tabular}

* Síndrome demencial: diagnóstico conforme critérios do DSM-IV (Diagnostic Statistical Manual of American Psychiatry Association, versão IV 6;

** MEEM: Mini-Exame do Estado Mental (pontos de corte: 18/19 - analfabetos; 23/24 - a partir de 1 ano de escolaridade) 26;

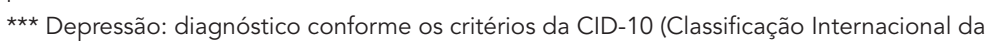
Doença - 10ạ revisão) 15 .

O processo de adaptação transcultural de um instrumento é uma atividade complexa, visto que envolve um conjunto de tarefas até que se alcance a equivalência funcional, um passo fundamental para reproduzi-lo no contexto cultural para o qual se pretende adaptá-lo. Importa conhecer as peculiaridades da amostra a ser investigada, levando-se em consideração que, quase sempre, determinado instrumento foi concebido para um tipo específico de população 28 .

Estes resultados, ainda que preliminares, mostraram que, entre a versão original e a versão do IQCODE-BR, as equivalências conceitual, de itens e semântica foram satisfatórias. De maneira geral, a escala se mostrou de fácil compreensão na amostra estudada. Ademais, se revelou um instrumento que, acompanhado de um manual, simplificado, com orientações padronizadas, 
Tabela 4

Manual de instruções resumido para aplicação da versão brasileira do Informant Questionnaire on Cognitive Decline in the Elderly (IQCODE-BR).

Item
1. Lembrar de rostos de parentes e amigos
2. Lembrar dos nomes de parentes e amigos
3. Lembrar de fatos relacionados a parentes e amigos
como, por exemplo: suas profissões, aniversários e endereços
4. Lembrar de acontecimentos recentes

4. Lembrar de acontecimentos recentes

5. Lembrar-se de conversas depois de poucos dias

6. No meio de uma conversa, esquecer o que ele(a) queria dizer

7. Lembrar do próprio endereço e telefone

8. Saber o dia e o mês em que estamos

9. Lembrar onde as coisas são geralmente guardadas

10. Lembrar onde encontrar coisas que foram guardadas em lugares diferentes daqueles em que costuma guardar

11. Adaptar-se a qualquer mudança no dia-a-dia

12. Saber utilizar aparelhos domésticos

13. Aprender a utilizar um novo aparelho existente na casa

14. Aprender coisas novas em geral

15. Lembrar das coisas que aconteceram na juventude

16. Lembrar de coisas que ele(a) aprendeu na juventude

17. Entender o significado de palavras pouco utilizadas

18. Entender o que é escrito em revistas e jornais

19. Acompanhar histórias em livros ou em programas de televisão

20. Escrever uma carta para amigos ou com fins profissionais

21. Conhecer importantes fatos históricos

22. Tomar decisões no dia-a-dia

23. Lidar com dinheiro para as compras

24. Lidar com assuntos financeiros, por exemplo: aposentadoria e conta bancária

\section{Significado}

Pessoas com as quais tenha contato

Pessoas com as quais tenha contato

Profissões ou locais em que trabalham, aniversários e onde moram

Qualquer evento importante que tenha acontecido nos últimos três meses (uma festa, nascimento ou morte de algum conhecido, viagem, hospitalização)

Qualquer conversa nos últimos três dias

Quando a pessoa está conversando, para e esquece o que estava dizendo e muda de assunto

Lembrar o nome da rua onde mora e telefone de sua residência

Esquecer freqüentemente o dia e o mês, sugerindo desorientação temporal Coisas que são colocadas sempre no mesmo lugar (por exemplo, as roupas e os talheres)

Coisas que não têm lugar fixo e, geralmente, são deixadas em locais diversos. Importante saber se isto acontece com freqüência (por exemplo, os óculos, as chaves, o dinheiro)

Quando ocorrem mudanças nas ações comumente realizadas, saber se o idoso se adapta com tranqüilidade e apresenta alterações de comportamento

Qualquer aparelho dentro de casa usado diariamente (por exemplo, liquidificador, ferro elétrico, fogão, gás)

Aparelho comprado recentemente que exija algum aprendizado para o uso (eletrodoméstico em geral) (algum aparelho novo que tenha comprado recente)

Capacidade de aprender a manusear algum aparelho ou executar uma nova receita

Lembrar onde trabalhou, com quem conviveu ou coisas que tenha feito quando jovem

Alguma atividade como consertos em casa ou qualquer equipamento. Atividades artesanais ou culinárias

Palavras que não são, corriqueiramente, utilizadas. Se ao se incluir uma palavra deste tipo em alguma conversa, o sentido deixa de ser entendido Ler e entender sobre o que o assunto se refere. Isto é percebido quando se tenta conversar sobre o que foi lido

Acompanhar a história do início ao fim sem perder o entendimento. Se consegue, por exemplo, assistir a uma novela, lembrando os detalhes do capítulo anterior

Escrever cartas ou preparar listas. Qualquer atividade que necessite fazer uso da escrita

Falar sobre fatos importantes como, por exemplo, quais foram os presidentes da república, ou fatos importantes que aconteceram neste ou em outros países

É autônomo para decidir quando vai ao médico ou quando vai comprar algo novo para casa

Manuseia dinheiro sabendo o valor que deve pagar e quanto de troco deve receber

Utiliza adequadamente sua conta bancária ou recebe sua aposentadoria fazendo uso correto do dinheiro

(continua) 
Tabela 4 (continuação)

25. Lidar com outros cálculos do dia-a-dia, por exemplo: quantidade de comida a comprar, Há quanto tempo não recebe visitas de parentes ou amigos

26. Usar sua inteligência para compreender e pensar sobre o que está acontecendo
Ao olhar para o armário de compras sabe o que está faltando. Sabe há quanto tempo, por exemplo, um filho não faz uma visita

Tem consciência do que está acontecendo ao seu redor. Quando está no médico, ou em uma festa, ou na casa de algum parente ou amigo pode ser administrado por profissional de saúde de várias categorias. Esta versão se encontra disponível na página da Internet http://cmhr. anu.edu.au/ageing/Iqcode/index.php, criada pelo Centro de Pesquisa em Saúde Mental da Austrália, para abrigar as diferentes versões do instrumento nos diversos idiomas para os quais foi adaptado.

No estudo realizado foram obtidos resultados positivos com a versão proposta para uso no
Brasil. No entanto, é possível que esta adaptação não apresente o mesmo desempenho em todos os países de língua portuguesa ou até mesmo em outras regiões brasileiras, dado que existe heterogeneidade entre a população. Provável que sejam necessários outros processos de adaptação que requeiram ajustes locais tendo em vista as peculiaridades regionais.

\section{Resumo}

O presente trabalho tem como objetivo apresentar os resultados do processo de tradução e adaptação transcultural do Informant Questionnaire on Cognitive Decline in the Elderly (IQCODE) e da confiabilidade teste-reteste de uma versão para uso no Brasil. Um comitê para revisão do instrumento analisou a equivalência conceitual e de itens. Para análise de equivalência semântica, foram realizadas três traduções e três retrotraduções; elaboração de uma versão síntese; préteste e elaboração de uma versão teste. A consistência interna e a confiabilidade teste-reteste foram mensu- radas pelo coeficiente $\alpha$ de Cronbach e o coeficiente de correlação intraclasse (CCI), respectivamente. Dos 169 informantes avaliados, 97 foram retestados. $\mathrm{O} \alpha$ de Cronbach foi 0,94 e o CCI foi de 0,92. Os níveis de confiabilidade obtidos permitem concluir que a versão do IQCODE-BR se mostrou de fácil compreensão, sendo observada uma boa equivalência com a versão original.

Questionários; Tradução; Demência 


\section{Colaboradores}

M. A. S. Sanchez contribuiu no planejamento, revisão bibliográfica, elaboração da versão teste do instrumento, seleção e treinamento dos auxiliares de pesquisa, organização e supervisão da todas as etapas de coleta de dados, supervisão do armazenamento de dados, tratamento estatístico e elaboração da versão final do manuscrito. R. A. Lourenço contribuiu com a orientação global do projeto elaboração da versão teste, revisão crítica do conteúdo e da versão final do manuscrito.

\section{Agradecimentos}

À equipe de profissionais do setor de Cuidado Integral à Pessoa Idosa da Universidade Aberta da Terceira Idade/Universidade do Estado do Rio de Janeiro. Aos integrantes do comitê de revisão do instrumento: Dra. Emylucy Paiva Paradela, Dra. Simone Garruth Sampaio, Dra. Irene Moreira e Dr. Tarso Morsi. Aos tradutores e retrotradutores do IQCODE: Dr. Sergio Telles Ribeiro Filho, Prof. Marco Antonio Ferreira de Souza, Prof. Ruthberg dos Santos, Prof. Jonh Wilkinson, Sr. Garry Oughton, Prof. Byron Shore e Prof. Marcelo Santos Afonso. Às auxiliares de pesquisa: Vânia Maria Silva de Moraes, Elizabeth Menezes, Roberta dos Santos, Flavia Rodrigues de Souza e Anik Setúbal. À supervisora de campo Dra. Silvia Merrhy Lagrota.

\section{Referências}

1. Scazufca M, Cerqueira ATR, Menezes PR, Prince M, Vallada HP, Miyazaki MCOS, et al. Investigações epidemiológicas sobre demência nos países em desenvolvimento. Rev Saúde Pública 2002; 36:773-8.

2. Scazufca M, Menezes PR, Vallada HP, Crepaldi AL, Pastor-Valero M, Coutinho LMS, et al. High prevalence of dementia among older adults from poor socioeconomic backgrounds in São Paulo, Brazil. Int Psychogeriatr 2008; 20:394-405.

3. Herrera Jr. E, Caramelli P, Silveira AS, Nitrini R. Epidemiologic survey of dementia in a communitydwelling Brazilian population. Alzheimer Dis Assoc Disord 2002; 16:103-8.

4. Bustamante SEZ, Bottino CM, Lopes MA, Azevedo D, Hototian SR, Litvoc J, et al. Instrumentos combinados na avaliação de demência em idosos: Resultados preliminares. Arq Neuropsiquiatr 2003; 61:601-6.

5. Instituto Brasileiro Geografia e Estatística. Pesquisa Nacional por Amostra de Domicílio - PNAD 2001. Composição e mobilidade nacional. http:// www.ibge.gov.br/home/estatistica/populacao/ perfilidoso/perfidosos2000.pdf (acessado em 10/ Mar/2009).
6. American Psychiatry Association. Diagnostic criteria from DSM-IV. Washington DC: American Psychiatry Association; 1994.

7. Folstein ME, Folstein SE, McHugh PR. Mini-mental state: a practical method for grading the cognitive state of patients for the clinician. J Psychiatr Res 1975; 12:189-98.

8. Bertolucci PH, Brucki SM, Campacci SR, Juliano Y. O mini-exame do estado mental em uma população geral: impacto da escolaridade. Arq Neuropsiquiatr 1994, 52:1-7.

9. Hototian S, Lopes MA, Bustamante SEZ, Azevedo D, Tatsch M, Litvoc J, et al. Identification of dementia suspects subjects in three districts of São Paulo city, Brazil. Int Psychogeriatr 2005; 17:271-2.

10. Lopes MA, Hototian SR, Bustamante SEZ, Azevedo D, Tatsch M, Bazzarella MC, et al. Prevalence of cognitive and functional impairment in a community sample in Ribeirão Preto, Brazil. Int J Geriatr Psychiatry 2007; 22:770-6.

11. Mackinnon A, Khalilian A, Jorm AF, Korten AE, Christensen H, Mulligan R, et al. Improving accuracy for dementia in a community sample by augmenting cognitive testing with informant report. J Clin Epidemiol 2003; 56:358-66. 
12. Mackinnon A, Mulligan R. Combining cognitive testing and informant report to increase accuracy in screening for dementia. Am J Psychiatry 1998; 155:1529-35.

13. Knafelc R, Lo Giudice D, Harrigan S, Cook R, Flicker L, Mackinnon A, et al. The combining of cognitive testing and a informant questionnaire in screening for dementia. Age Ageing 2003; 32:541-7.

14. McKhann G, Drachaman D, Folstein M, Katzman R, Price D, Stadian E. Clinical diagnosis of Alzheimer's disease: report of the NINCDS-ADRDA Work Group under the auspices of Department of Health and Human Services Task Force of Alzheimer's disease. Neurology 1984; 34:939-44.

15. World Health Organization. The ICD 10 classification of mental and behavioral disorders. Geneva: World Health Organization; 1992.

16. Jorm AF. The Informant Questionnaire on Cognitive Decline in the Elderly (IQCODE): a review. Int Psychogeriatr 2004; 16:1-19.

17. Mulligan R, Mackinnon A, Jorm AF, Giannakopoulos P, Michel JPA. Comparison of alternative methods of screening for dementia in clinical settings. Arch Neurol 1996; 532-6.

18. Jorm AF. Methods of screening for dementia: a meta-analysis of studies comparing an informant questionnaire with a brief cognitive test. Alzheimer Dis Assoc Disord 1997; 11:158-62.

19. Morales JM, Bermejo F, Romero M, Del-Ser T. Screening of dementia in community-dwelling elderly through informant report. Int J Ger Psychiatry $1997 ; 12: 808-16$.

20. Jorm AF, Broe GA, Creasey H, Sulway MR, Dente O, Fairley MJ, et al. Further data on the validity of the Informant Questionnaire on Cognitive Decline in the Elderly (IQCODE). Int J Ger Psychiatry 1996; 11:131-9.

21. Jorm AF, Korten AE. Assessment of cognitive decline in the elderly by informant interview. Br J Psychiatry 1988; 152:209-13.
22. Jorm AF. A short form of the Informant Questionnaire on Cognitive Decline in the Elderly (IQCODE): development and cross-validation. Psychol Med 1994; 24:145-53.

23. Thomas LD, Gonzales MF, Chamberlain A, Beyreuther K, Masters CL, Flicker L. Comparison of clinical state, retrospective informant interview and the neuropathologic diagnosis of Alzheimer's disease. Int J Ger Psychiatry 1994; 9:233-6.

24. Jorm AF, Scott R, Jacomb PA. Assessment of cognitive decline in dementia by informant questionnaire. Int J Ger Psychiatry 1989; 4:35-9.

25. Jorm AF, Jacomb PA. The Informant Questionnaire on Cognitive Decline in the Elderly (IQCODE): socio-demographic correlates, reliability and some norms. Psychol Med 1989; 19:1015-22.

26. Lourenço RA, Veras RP. Mini-Exame do Estado Mental: características psicométricas em idosos ambulatoriais. Rev Saúde Pública 2006; 40:712-9.

27. Herdman M, Fox-Rushby J, Badia X. A model of equivalence in the cultural adaptation of HRQoL instruments: the universalist approach. Qual Life Res 1998; 7:323-35.

28. Reichenheim ME, Moraes CL. Operacionalização de adaptação transcultural de instrumentos de aferição usados em epidemiologia. Rev Saúde Pública 2007; 41:665-73.

29. Reichenheim ME, Moraes CL. Buscando a qualidade das informações em pesquisas epidemiológicas. In: Minayo MCS, Deslandes SF, organizadoras. Caminhos do pensamento: epistemologia e método. Rio de Janeiro: Editora Fiocruz; 2002. p. 227-54.

30. Fuh JL, Teng EL, Lin KN, Larson EB, Wang SJ, Liu CY, et al. The Informant Questionnaire on Cognitive Decline in the Elderly (IQCODE) as a screening tool for dementia for a predominantly illiterate Chinese population. Neurology 1995; 45:92-6.

31. Tang WK, Chan SSM, Chiu HFK, Wong KS, Kwok TCY, Mok V, et al. Can IQCODE detect poststroke dementia? Int J Ger Psychiatry 2003; 18:706-10.

Recebido em 10/Nov/2008

Versão final reapresentada em 13/Jan/2009 Aprovado em 04/Mar/2009 